\title{
The Development of Cancer Genome Research and its Clinical Opportunity brought by Next Generation Sequencing
}

Jun Li, Bin Wang, Hu Fang and Xu Yang*

Department of Human Medical Research, Research \& Cooperation Division, BGI-Shenzhen, China

\begin{abstract}
Cancer has been investigated due to its high mortality ever since last century. Although chemotherapy has been traditionally used for cancer patients for a long time, the obvious side effects have prevented its further application. With the progress of DNA sequencing technology, targeted therapy has been developed by affecting targeted molecules in the signaling pathways. It could act as an alternative strategy of cancer therapeutics. In this way, cancer patients could undergo fewer side effects than traditional chemotherapy. In the $21^{\text {st }}$ century, next generation sequencing (NGS) technologies have emerged to dramatically promote the cancer genome research. Recent researches have shown that the rapid discoveries of mutated cancer genes by NGS are potential to revolutionize cancer therapeutics. In the future, the development of NGS technologies is hopeful to achieve personalized therapeutics in clinic.
\end{abstract}

Keywords: Cancer genome; Targeted therapy; Signaling pathway; Clinical application; Next generation sequencing; Personalized therapeutics

\section{Introduction}

Cancer accounts for the most mortality in human diseases. It is a tremendous disaster for human beings. Thus, scientists all around the world have continuously devoted efforts to cancer research in order to find effective therapy from the beginning of last century. Chemotherapy has been used as the traditional cancer therapy for a long time. In the 1940s, the era of chemotherapy began by using nitrogen mustards and antifolate as drugs [1]. Even now, chemotherapy is still widely used for cancer patients. The mechanism of traditional chemotherapy is to inhibit the division of cancer cells by chemotherapy drugs. However, certain normal cells with rapid division would be inevitably influenced by chemotherapy, like hair, gastrointestinal epithelium, and bone marrow cells. Thus, many patients suffered from the consequent side effects, typically alopecia, gastrointestinal symptoms, and myelosuppression [2]. With the development of cancer research, the strategy of cancer therapy has shift sharply in the past decades. Together with the identification of DNA as the inherited material and the emergence of sequencing technology, targeted therapy of cancer genes has gradually become the optimal strategy of cancer therapeutics, such as breast, colorectal, lung, and pancreatic cancers, as well as lymphoma, leukemia, and multiple myeloma [2]. It is reported that more than $1 \%$ of genes contribute to human cancer [3]. However, the number of cancer genes is underestimated primarily due to the low-throughput sequencing technology. The whole cancer genome profiling is essential for the understanding of tumorigenesis and would also provide insight into target therapy. High-throughput sequencing technology is expected to decode the cancer genome. In recent years, the advent of next generation sequencing (NGS) technologies has greatly contributed to the achievement of the goal.

As a powerful tool, the advent of NGS technologies has obviously accelerated the research of cancer genome. Benefited from highthroughput advantage, rapid developments of genome-wide sequencing technologies and computational bioinformatics have contributed to the improvement of cancer research. The genetic codes can be mapped and studied at the whole genome level, instead of a certain genome region. This provides us new insights into diagnosis, prognosis and treatment of cancer. However, the massive amount of sequencing data has revealed that the complexity of cancer heterogeneity is far beyond our expectation. The result is different from our previous thought. Thus, we cannot directly use abundant information of cancer genomics for cancer medical applications. There is an invisible but huge gap between the scientific discovery and the clinical application.

The gap is caused by the slow progress of translation from cancer research discovery to clinical use. There are two main reasons. First, the mainstream research tools of various assays lead to an incomplete catalog of various mutations in cancer genome. Therefore, the researchers' view is limited to certain gene families or pathways. Researchers can not have the integrated insight based on whole genome scale. Thus, the explanations of mutations are possibly not comprehensive or correct. The complete map of cancer genome is extremely necessary for systematic cancer research at genome-wide level. Second, various mutations are not clearly understood for their roles in cancer development. Cancer exhibits the unique characteristic of heterogeneity, which is a big challenge for scientific research and translation. It means that the activities of oncogenes depend on cell type, tissue type, developmental stage and individual condition. Thus, it is tough to know how each causative gene contributes to the development and maintenance of cancer. This high complexity is a great obstacle for clinical application of cancer research. Accordingly, limited scientific discoveries can be applied for patients with the same cancer type. For a certain cancer type, at least hundreds of samples are required to explore the highly complex nature of cancer. NGS technologies have served as the engine to promote the clinical translation, and the gap is being covered by continuous progress in cancer genomics.

*Corresponding author: Xu Yang, Ph.D, Director, Department of Human Medical Research, Research \& Cooperation Division, BGI-Shenzhen, China, Tel: +8675525273528/8615889620343; Fax: +86755 25274183; E-mail: yangxu@genomics.cn

Received Novmeber 23, 2011; Accepted January 30, 2012; Published February 06, 2012

Citation: Li J, Wang B, Fang H, Yang X (2012) The Development of Cancer Genome Research and its Clinical Opportunity brought by Next Generation Sequencing. J Health Med Informat S1. doi:10.4172/2157-7420.S1-002

Copyright: (c) $2012 \mathrm{Li}$ J, et al. This is an open-access article distributed unde the terms of the Creative Commons Attribution License, which permits unrestricted use, distribution, and reproduction in any medium, provided the original author and source are credited. 
In this paper, we will first review the previous techniques and achievements of cancer research before the advent of NGS technologies. Then, recent advances in NGS technologies will be highlighted to demonstrate the potential contribution of cancer genomics in the future for prevention, detection and treatment.

\section{Cancer Research before NGS Technologies}

\section{Traditional cytogenetic study}

During the cancer research in the past several decades, a variety of techniques were applied to investigate various cancer types. In the earliest stage of cancer research, the chromosome cytogenetic study of cancer cells was the mainstream method. By observing a population of cancer cells, scientists had found that cancer cells appeared to be apparently more unstable than normal cells. Thus, it was hypothesized that the tumor progression might result from acquired genetic variability within the original clone. These researches demonstrated the cytogenetic heterogeneity in human malignancies, suggesting that each patient may require personalized diagnosis and treatment [4].

According to the low resolution of cytogenetic study, chromosome was the preferred objective in cancer research. In 1960, the chromosome translocation between chromosomes 9 and 22 was revealed in Chronic Myeloid Leukemia (CML) cells, leading to BCR-ABL fusion gene [5]. Four decades later, the BCR-ABL fusion is regarded as the effective drug target to treat CML patients. In a word, traditional cytogenetic study had demonstrated the cytogenetic heterogeneity of cancer cells and the phenomenon of chromosomal translocation. Moreover, the type and extent of cancer cell aberrations were suggested to be associated with tumorigenesis. Thus, insights had been offered into cancer research that laid the basis for subsequent research and indicated the developmental direction in the future.

\section{PCR-based direct sequencing study}

Based on the development of cytogenetic techniques in recent years, the resolution of cancer genome research continuously increased. In the $20^{\text {th }}$ century, it was available to focus on the aberrant genomic regions. Thus, not only recurrent chromosomal rearrangements, but the first cancer gene was identified. In the 1980s, HRAS was identified as the first cancer gene, a point mutation at codon 12 in HRAS responsible for activation of cancer development [6,7]. The oncogenic mutation could induce the change from glycine to valine. Subsequently, two members of RAS family were identified in the next few years. KRAS [810] and NRAS [11] were found to be activated in human tumors. Since then, RAS family genes had been the hotspot of cancer research. They are a set of several similar genes with similar biochemical functions. RAS family genes could encode small proteins with enzymatic GTPase activity, generally responsible for cell signal transduction. The signaling activation by RAS family would lead to cell proliferation and survival, which would function as regulatory switches governing a variety of cellular behaviours. Thus, the dysregulation of RAS signaling pathways was one of the main foundations of tumorigenesis. The activation mutations of RAS family genes had been found in various human cancers, such as colon, kidney, liver cancers. Furthermore, the frequency was especially high in specific tumor types. For example, it was observed that $90 \%$ of pancreatic cancer harbored the KRAS mutation [12]. In conclusion, the important roles of three cancer genes, consisting of HRAS, KRAS and NRAS, had attracted the attention of cancer researchers.
Even though the significance of cancer gene has been acknowledged, it may be still underestimated due to the limitation of techniques in the $20^{\text {th }}$ century. First, the resolution of methods then was not high enough to identify small-scale variants. Hence, the identification of genomewide base substitution and small indels was not available for previous methods. Second, the identification was based on the primer design of Polymerase Chain Reaction (PCR) reaction to amplify interested sequences for analysis. Before the accomplishment of Human Genome Project (HGP), there was no comprehensive template for PCRbased cancer research. However, the lack was covered along with the accomplishment of human genome draft map in 2000, which served as the perfect reference. After that, extensive research by targeted sequencing of candidate genes had been conducted in the field of cancer genomics, leading to the identification of some cancer genes as drug targets. For example, a recurrent point mutation in BRAF could result in the substitution from glutamic acid to valine at residue 600 . The mutation was found to be closely associated with tumorigenesis and highly prevalent in malignant melanomas [13]. BRAF is a central transduction mediator in the RAS-RAF-MEK-ERK-MAP signaling pathway. Binding to RAS proteins, BRAF could transfer the upstream growth factor signals through its phosphylation and the subsequent phosphylation of downstream MEK proteins. The highly prevalent and recurrent characteristics of BRAF mutation in melanomas make it suitable for therapeutic target [14]. In 2010, eight years after its discovery, mutant BRAF inhibitors have been preliminarily translated into personalized medicine. PLX4032 (also known as vemurafenib or zelboraf) exhibits extraordinarily good effects in the stage of clinical trials. This is relatively rapid translation from cancer research discovery to targeted therapy.

\section{Targeted cancer therapy}

Along with the progress of PCR-based direct sequencing and pathway analysis, targeted therapy has dramatically improved cancer therapeutics in the past more than one decade. Different from traditional cytotoxic chemotherapy, the mechanism of targeted therapy is to block the propagation of cancer cells. It is achieved by the interference with targeted molecules in certain signaling pathways. These targeted molecules are responsible for cell proliferation and over expressed in normal cells. According to current research, these pathways with the enrichment of targeted molecules mainly exist in solid tumors, such as lung, colorectal, and breast cancers. Furthermore, human epidermal growth factor receptor (HER1 or EGFR), vascular endothelial growth factor (VEGF) and HER2 are frequently reported among targeted molecules in the pathways $[15,16]$. The inhibition can be guided by blocking the binding of ligand and receptor or by the interference with downstream molecules [2]. As the mechanism described above, targeted therapies could try to avoid destroying the normal cells as much as possible. Thus, patients could suffer fewer side effects than traditional chemotherapy, such as cardiac dysfunction and proteinuria. Since 2000, U.S. Food and Drug Administration (FDA) has approved much more drugs of targeted cancer therapies than those of traditional chemotherapy.

Here, a few examples of targeted cancer therapy will be demonstrated. As mentioned above, RAS family had been involved in extensive cancer research in the $20^{\text {th }}$ century, especially HRAS, KRAS and NRAS. These three RAS genes were found to regulate the GTPase activity of RAS family, contributing to prevent GAP from promoting the hydrolysis of GTP on RAS. Among them, KRAS mutation (about 
85\%) was frequently found in tumors, with less frequency of NRAS (about 15\%) and HRAS mutations (about 1\%) [12]. With the clear understanding of its characteristics, KRAS is expected to be the most potential member in RAS family for clinical translation as a effective target. In the $21^{\text {st }}$ century, the accomplishment of HGP is the milestone for human research in every aspect, including human cancer research. Since it can provide the genome with single base resolution, HGP has made great contribution to cancer research. KRAS and BRAF are representative examples of targeted cancer therapy. In 2000, BRAF was discovered to be a potential new therapeutic target through a systematic genome-wide sequencing screen [13]. BRAF mutations were found to be mutated in $66 \%$ of malignant melanomas, but with the lower frequency in other cancers. A single substitution (V600E) in the BRAF accounted for $80 \%$ of all mutations. BRAF encodes a serine/ threonine kinase, while mutated BRAF proteins exhibit the elevated activity of kinase. As a downstream mediator in the RAS-RAF-MEKERK-MAP pathway, mutant BRAF could enable the proliferation of cancer cells without RAS function. Recently, V600E mutant inhibitors of BRAF were reported to be in phase 1 trials, showing good responses for metastatic malignant melanomas with V600E mutations [17]. Although recurrence is found after BRAF inhibitors therapy, further exploration has revealed some recurrence-associated mutations as potential therapeutic targets $[18,19]$.

In addition to the discovery of BRAF as the therapeutic target, the mechanism and role of KRAS discovered in 1983 are also increasingly clear. KRAS is reported to be the downstream signaling mediator of EGFR in the EGFR-KRAS-BRAF signaling pathway. KRAS and BRAF in wild type could not transduce the growth signal. Thus, the proliferation, survival and metastasis of cancer cells are blocked. Oppositely, mutant KRAS and BRAF would activate the signaling pathway. KRAS is currently treated as a marker of clinical testing for patients with lung or metastatic colorectal cancer. It is used to evaluate the response to anti-epidermal growth factor receptor (EGFR) therapy. The choice of drug in EGFR-targeted therapy is dependent on the result of KRAS testing, which serves as a predictive and prognostic indicator [20-23]. For example, for metastatic colorectal cancer with EGFR mutation, cetuximab and panitumumab are totally useless for mutantKRAS patients. However, they exhibit higher treatment rate and longer survival time for wild-KRAS patients $[21,22]$. Likewise, for non-small cell lung carcinoma (NSCLC), the effect of erlotinib is better for wildKRAS patients than mutant-KRAS ones [23]. RAS family genes are involved in different pathways. And frequent mutations of them are found in human cancers. Thus, RAS family genes are regarded as the crucial contributor in tumorigenesis, as well as the targets of many anti-cancer therapies.

Along with technique advances in the $21^{\text {st }}$ century, research methods show higher resolution than previous cytogenetic method. In this way, many novel cancer genes have been detected by investigating in the regions with recurrent copy number variation [3,24]. Furthermore, gene fusions caused by chromosomal rearrangements have been identified to be more than 300 [25]. Gene fusions are found widely distributed in human malignancies, not only hematopoietic malignancies [26], but also some solid tumors, e.g. lung and prostate cancers $[27,28]$. It is reported that gene fusions are presented in all malignancies, accounting for $20 \%$ of human cancer morbidity [25]. According to the high prevalence, gene fusions have been recognized as a diagnostic and prognostic indicator. It is proven by accumulated evidence in the initiation stage of tumorigenesis. Imatinib (also known as Gleevec or Glivec) is an excellent example to illustrate the role of gene fusion in targeted cancer therapy. As one of the specific inhibitors in targeted therapy, imatinib has been approved by FDA in 2001 to effectively treat CML by inhibiting BCR-ABL tyrosine kinase [29]. BCR-ABL is originated from Philadelphia chromosome translocation that fuses BCR sequences on chromosome 22 to $\mathrm{ABL}$ genes on chromosome 9 [5]. Since the chromosomal abnormality was found to be present in almost all CML patients, $98 \%$ patients showed completely good hematologic response to imatinib therapy [30,31]. In 2002, imatinib has also been approved by FDA to treat gastrointestinal stromal tumors (GISTs). Besides BCR-ABL, imatinib could also inhibit other targets, e.g. c-kit and Platelet-Derived Growth Factor Receptor (PDGF-R). Now, imatinib has been used for several kinds of cancers and diseases, e.g. hyper eosinophilic syndrome and dermatofibrosarcoma protuberans [32].

In addition to drug targets mentioned above, the effectiveness of targeted therapy relies on drug dose. Drug overdose could lead to toxicity. Thus it is partially responsible for the side effects of targeted therapy. It is reported that optimal drug dose can be determined by assessing the dynamics of pharmaceutical treatment, e.g. the levels of circulating tumor cells and endothelial cells or the serial level of target molecules in tumor tissue [2,33-35]. How to choose the drug dose of targeted therapy is a challenge for cancer researchers. It is expected to be solved with the advances of technologies in the future.

\section{Cancer pathway study}

A large set of candidate cancer genes (CAN genes) have been identified based on previous researches. And there is heterogeneity for CAN genes. CAN genes would even differ between two tumors within the same type. However, the pathway analysis so far has suggested that signaling pathways are more responsible for tumorigenesis than individual cancer gene. Although many CAN genes have been revealed in the past decades, the number of dysregulated signaling pathways is reported to be limited in cancers [36]. The variety of CAN genes could function through the few signaling pathways. For example, the aberration of WNT signaling pathway is found to be associated with colorectal cancer as well as breast cancer. However, the causative cancer genes are diverse in the two cancer types. Specifically, the adenomatous polyposis of the colon (APC) gene in the WNT pathway could induce colorectal cancer with high frequency of mutations. In breast cancer, WNT pathway is activated by the stabilization of $\beta$-catenin that is a downstream component of WNT pathway [37]. The phenomenon indicates the importance of signaling pathways in the contribution to tumorigenesis. Mutations in any component of signaling pathways would lead to the similar dysregulation effects for tumorigenesis.

The example of WNT pathway in both colorectal and breast cancers mentioned above demonstrates the involvement of one signaling pathway in several cancer types. Since many CAN genes are enriched in the signaling pathways, the tumorigenesis of various cancer types is often induced by the dysregulation of common signaling pathways. For example, it is revealed that several dysregulated signaling pathways in glioblastoma multiforme are also associated with pancreatic, colorectal and breast cancers. In addition to the common pathways, a few specific pathways in the glioblastoma multiforme would act as ion channels in nervous system-specific cellular pathways. However, the proliferation of cancer cells in these cancer types are mainly controlled by the common pathways, which are responsible for cellular growth, apoptosis and adhesion [38,39]. Thus, it seems that the dysregulated signaling pathways rather than individual cancer gene would dominate 
the tumorigenesis. The inspiration has prompted the researchers to focus on signaling pathways, rather than individual mutant genes.

Generally, only the genome-wide screening of cancer genome can identify all CAN genes in a certain cancer type. Thus, the information of genome-wide screening is required to analyze the contribution of pathways to tumorigenesis. Although the achievement of HGP has significantly improved the cancer research, the high cost and low capacity of direct sequencing technology in the $21^{\text {st }}$ beginning cannot meet the requirement of cancer pathway study. Thus, high-throughput NGS is critical to decode the genomes of various cancer types and analyze the oncogenic signaling pathways.

\section{Cancer Research with NGS Technologies}

\section{Next generation sequencing era}

The appearance of numerous cancer patients each year requires a time and cost-effective method for clinical application. The problem is expected to be solved with the advent of NGS technologies, due to the advantages of massively parallel sequencing and high-throughput data output. In 2008, the first comprehensive cancer genome was generated through whole geonome sequencing (WGS) of acute myeloid leukaemia (AML) [40]. This suggests that cancer genome research has entered into the NGS era. By sequencing the genomic DNA of a typical AML-M1 tumor and the matched normal skin from the same patient, ten genes with acquired mutations were discovered. Among them, eight SNVs were new mutations in almost all tumor cells at present and relapse 11 months later. The research revealed a single dominant clone in the patient including all the mutations, and demonstrated the power of NGS to reveal novel genes that might respond to targeted therapies. Inspired by the above results, several whole genome sequencing researches on AML were subsequently conducted. And these researches had led to the discovery of novel isocitrate dehydrogenase 1 (IDH1), isocitrate dehydrogenase 2 (IDH2), DNA methyltransferase 3a (DNMT3A) and TP53 mutations as AML-associated cancer genes [41-44]. However, the clinical features of these mutations remained unclear. Then, other researchers continued to investigate their prognostic impact on AML [45-47]. It was found that IDH1 and IDH2 mutations were poor prognostic factors for cytogenetically normal AML (CN-AML) patients $[45,46]$. But, the screening of IDH1 mutations could be used for risk stratification to identify the CN-AML patients with high-risk [47]. The results of researches on the same cancer type suggest that the known cancer genes are limited. And more causative cancer genes still remain to be discovered than those known ones.

With the wide application of NGS technologies, a series of cancer genome researches has been conducted on many other cancer types, such as colorectal cancer, lung cancer, breast cancer, hepatocellular carcinoma, etc (Table 1). The comprehensive information of the largescale cancer genome sequencing has revealed the catalogue of cancer mutations, which would lead the diagnosis, prognosis and treatment of cancer into the NGS era.

\section{The catalogue of cancer mutations}

These foregoing cancer genome researches have revealed the comprehensive catalogue of somatic mutations in individual cancer genome, including single nucleotide variation (SNV), indels, copy number variation (CNV) and structural variation (SV) [48]. As the cost of NGS decreases dramatically in recent years, thousands of whole cancer genomes have already been sequenced. These comprehensive researches of diverse cancer genomes have offered us the first insight that the landscape of somatic mutations could be categorized into driver and passenger mutations [49]. Driver mutations could confer the growth advantage to cancer cells, allowing them to be out of control in the cell proliferation, differentiation and apoptosis. Thus, driver mutations can enable cancer cells to grow in primary tissues and metastasize to the surrounding tissues. Although the cancer in the final stage may not carry them, driver mutations have absolutely played an important role in the cancer development. And the rest mutations are passenger mutations, conferring no growth advantage to cancer cells. So far, the majority of mutations in individual cancer genome are passenger mutations [50-53]. From the view of evolution, cancer would arise as the accumulated driver mutations in cancer cell clones with cell division [48].

Due to the central role of driver mutations in tumorigenesis, the key challenge is to distinguish the type and number of novel driver mutations from the numerous mutations in the cancer genome research by NGS. On one hand, there are other types of mutations except common point mutations. For example, the large-scale structural variations induced by chromosomal rearrangements remain relatively unfrequent and unexplored. Although the presence of chromosomal abnormality has been revealed by early cytogenetic study before [5], the prospect of chromosomal rearrangements has gradually become clear by the recent NGS-based cancer genome researches [54]. Many somatically acquired genomic rearrangements are found in human cancer genome. They have obviously distinct pattern from germline rearrangements. On the other hand, individual cancer differs in the number of mutations. For point mutation, some cancer genomes have more than 100,000 while fewer than 1,000 reside in others. For somatically acquired rearrangements, some cancer genomes exhibit numerous while others contain only few [48]. Now, it is unable to explain the pattern of somatic mutations with low frequency. But, another pattern of highly prevalent somatic mutations is reported to be probably caused by exposure to environmental carcinogens, as well as DNA repair defects [49]. For example, ultraviolet light exposure has been recognized to enhance the risk of malignant melanoma, while tobacco carcinogens can induce lung cancer [51,52]. Other mechanisms underlying the opposite patterns of somatic mutations in human cancers still remain to be investigated.

The solution to solve the challenge of identifying driver mutation is dependent on the integration of bioinformatic analysis and large sample size. Although NGS technologies have enabled the discovery of many driver mutations in cancer genes, most of them are lowfrequency mutations with no significance in clinical use. Due to the heterogeneity of cancer, at least hundreds of samples are required to identify cancer genes as the effective biomarkers. They might be used for clinical application on a particular cancer type. Meanwhile, the large-scale cancer genome research will generate a mass of sequencing data to deal with. Thus, strong bioinformatics software is required to filter errors with unbiased strategies to utilize useful sequencing data. Then, it is necessary to conduct the follow-up functional study of the identified cancer genes. So they could be confidently used for clinical application. In general, extensive efforts must be done to identify the effective driver mutations in clinic.

\section{Somatic mutations in metastases}

Metastasis refers to the fact that tumors would be found in other organs beyond primary tumor tissue ever since years of diagnosis. As an extremely complex process, it is always a major obstacle in cancer 
Citation: Li J, Wang B, Fang H, Yang X (2012) The Development of Cancer Genome Research and its Clinical Opportunity brought by Next Generation Sequencing. J Health Med Informat S1. doi:10.4172/2157-7420.S1-002

\begin{tabular}{|c|c|}
\hline Cancer Type & Literature \\
\hline Colorectal Cancer & $\begin{array}{l}\text { Adam J Bass, Michael S Lawrence, Lear E Brace, Alex H Ramos, Yotam Drier,. et al. (2011) Genomic sequencing of colorectal } \\
\text { adenocarcinomas identifies a recurrent VTI1A-TCF7L2 fusion. Nature genetics 43: 964-968. } \\
\text { De Grassi A, Segala C, lannelli F, Volorio S, Bertario L, et al. (2010) Ultradeep sequencing of a human ultraconserved region reveals } \\
\text { somatic and constitutional genomic instability. PLoS Biology } 8(1) \text { : e1000275. } \\
\text { Tie J, Lipton L, Desai J, Gibbs P, Jorissen RN, et al. (2011) KRAS mutation is associated with lung metastasis in patients with curatively } \\
\text { resected colorectal cancer. Clin Cancer Res 17(5): 1122-1130. }\end{array}$ \\
\hline Lung Cancer & $\begin{array}{l}\text { Pleasance ED, Stephens PJ, O'Meara S, McBride DJ, Meynert A, et al. (2010) A small-cell lung cancer genome with complex signatures } \\
\text { of tobacco exposure. Nature 463(7278): } 184-190 . \\
\text { Lee W, Jiang Z, Liu J, Haverty PM, Guan Y, et al. (2010) The mutation spectrum revealed by paired genome sequences from a lung } \\
\text { cancer patient. Nature 465(7297): 473-477. }\end{array}$ \\
\hline Hepatocellular Carcinoma & $\begin{array}{l}\text { Tao Y, Ruan J, Yeh SH, Lu X, Wang Y, et al. (2011) Rapid growth of a hepatocellular carcinoma and the driving mutations revealed by } \\
\text { cell-population genetic analysis of whole-genome data. Proceedings of the National Academy of Sciences 108(29): } 12042-12047 . \\
\text { Li M, Zhao H, Zhang X, Wood LD, Anders RA, et al. (2011) Inactivating mutations of the chromatin remodeling gene ARID2 in } \\
\text { hepatocellular carcinoma. Nature genetics 43(9): 828-829. } \\
\text { Totoki Y, Tatsuno K, Yamamoto S, Arai Y, Hosoda F, et al. (2011) High-resolution characterization of a hepatocellular carcinoma genome. } \\
\text { Nat Genet 43(5): 464-469. }\end{array}$ \\
\hline Multiple Myeloma & $\begin{array}{l}\text { Chapman MA, Lawrence MS, Keats JJ, Cibulskis K, Sougnez C, et al. (2011) Initial genome sequencing and analysis of multiple } \\
\text { myeloma. Nature 471(7339): 467-472. }\end{array}$ \\
\hline Melanoma & $\begin{array}{l}\text { Wei X, Walia V, Lin JC, Teer JK, Prickett TD, et al. (2011) Exome sequencing identifies GRIN2A as frequently mutated in melanoma. } \\
\text { Nature genetics } 43(5) \text { : 442-446. }\end{array}$ \\
\hline Lymphoma & $\begin{array}{l}\text { Pasqualucci L, Dominguez-Sola D, Chiarenza A, Fabbri G, Grunn A, et al. (2011) Inactivating mutations of acetyltransferase genes in } \\
\text { B-cell lymphoma. Nature 471(7337): 189-195. } \\
\text { Morin RD, Mendez-Lago M, Mungall AJ, Goya R, Mungall KL, et al. (2011) Frequent mutation of histone-modifying genes in non-Hodgkin } \\
\text { lymphoma. Nature 476(7360): 298-303. } \\
\text { Morin RD, Johnson NA, Severson TM, Mungall AJ, An J, et al. (2010) Somatic mutations altering EZH2 (Tyr641) in follicular and diffuse } \\
\text { large B-cell lymphomas of germinal-center origin. Nat Genet 42(2): 181-185. }\end{array}$ \\
\hline Prostate Cancer & $\begin{array}{l}\text { Kumar A, White TA, MacKenzie AP, Clegg N, Lee C, et al. (2011) Exome sequencing identifies a spectrum of mutation frequencies in } \\
\text { advanced and lethal prostate cancers. Proceedings of the National Academy of Sciences 108(41): 17087-17092. } \\
\text { Berger MF, Lawrence MS, Demichelis F, Drier Y, Cibulskis K, et al. (2011) The genomic complexity of primary human prostate cancer. } \\
\text { Nature 470(7333): } 214-220 .\end{array}$ \\
\hline Breast Cancer & $\begin{array}{l}\text { Inaki K, Hillmer AM, Ukil L, Yao F, Woo XY, et al. (2011) Transcriptional consequences of genomic structural aberrations in breast cancer. } \\
\text { Genome Research 21(5): } 676-87 \text {. } \\
\text { Walsh T, Lee MK, Casadei S, Thornton AM, Stray SM, et al. (2010) Detection of inherited mutations for breast and ovarian cancer using } \\
\text { genomic capture and massively parallel sequencing. Proceedings of the National Academy of Sciences } 107(28): 12629-12633 . \\
\text { Ding L, Ellis MJ, Li S, Larson DE, Chen K, et al. (2010) Genome remodelling in a basal-like breast cancer metastasis and xenograft. } \\
\text { Nature 464(7291): 999-1005. }\end{array}$ \\
\hline Renal Carcinoma & $\begin{array}{l}\text { Varela I, Tarpey P, Raine K, Huang D, Ong CK, et al. (2011) Exome sequencing identifies frequent mutation of the SWI/SNF complex } \\
\text { gene PBRM1 in renal carcinoma. Nature } 469(7331): 539-542 \text {. } \\
\text { Dalgliesh GL, Furge K, Greenman C, Chen L, Bignell G, et al. (2010) Systematic sequencing of renal carcinoma reveals inactivation of } \\
\text { histone modifying genes. Nature 463(7279): } 360-363 \text {. }\end{array}$ \\
\hline Gastric Cancer & $\begin{array}{l}\text { Holbrook JD, Parker JS, Gallagher KT, Halsey WS, Hughes AM, et al. (2011) Deep sequencing of gastric carcinoma reveals somatic } \\
\text { mutations relevant to personalized medicine. J Transl Med 9:119. } \\
\text { Zang ZJ, Ong CK, Cutcutache I, Yu W, Zhang SL, et al. (2011) Genetic and Structural Variation in the Gastric Cancer Kinome Revealed } \\
\text { through Targeted Deep Sequencing. Cancer research 71(1): 29-39. }\end{array}$ \\
\hline Pancreatic Cancer & $\begin{array}{l}\text { Jiao Y, Shi C, Edil BH, de Wilde RF, Klimstra DS, et al. (2011) DAXX/ATRX, MEN1, and mTOR pathway genes are frequently altered in } \\
\text { pancreatic neuroendocrine tumors. Science 331(6021): 1199-1203. } \\
\text { Campbell PJ, Yachida S, Mudie LJ, Stephens PJ, Pleasance ED, et al. (2010) The patterns and dynamics of genomic instability in } \\
\text { metastatic pancreatic cancer. Nature 467(7319): 1109-1113. } \\
\text { Yachida S, Jones S, Bozic I, Antal T, Leary R, et al. (2010) Distant metastasis occurs late during the genetic evolution of pancreatic } \\
\text { cancer. Nature 467(7319): 1114-1117. }\end{array}$ \\
\hline Leukemia & $\begin{array}{l}\text { Link DC, Schuettpelz LG, Shen D, Wang J, Walter MJ, et al. (2011) Identification of a novel TP53 cancer susceptibility mutation } \\
\text { through whole-genome sequencing of a patient with therapy-related AML. JAMA 305(15): 1568-1576. } \\
\text { Ley TJ, Ding L, Walter MJ, McLellan MD, Lamprecht T, et al. (2010) DNMT3A mutations in acute myeloid leukemia. N Engl J Med } \\
\text { 363(25): } 2424-2433 \text {. }\end{array}$ \\
\hline
\end{tabular}

Table 1: The list of some cancer genome researches by NGS application on many cancer types since 2010. 
therapy. According to cancer genome research, metastasis is originated from somatic mutations in the primary cancer cell clones under the selective pressures of microenvironment [48]. When the cancer cell clones continue to disseminate and adapt to the microenvironment of a distant tissue, the minority of cancer cells would colonize to the metastatic site. During the process of clonal expansion, a driver mutation from the primary cancer cell clone will establish the dominant subclone. Then, the dominant subclone would further form a minor subclone containing another driver mutation. The emerging driver mutation from the minor subclone would continually give rise to the next dominant subclone (Figure 1). Thus, there is a complex evolutionary history in the cancer with multiple clonal expansions.

By analyzing driver mutations along the process of clonal expansion, it is able to reconstruct the evolutionary tree of individual cancer from complex subclonal structure [55]. This is confirmed by a recent cancer research based on NGS. Compared with primary basallike breast tumour, the differential frequencies of mutations, as well as the patterns of structural variations, in metastasis and xenograft have been revealed. The result further indicates the distinct characterization of metastatic tumour [56]. For colorectal cancer, KRAS mutations seem to be associated with lung metastasis in patients [57]. These findings highlight somatic mutations as the potential biomarker to treat the metastatic cancers. However, the clinical application still requires extensive researches. The advent of single cell sequencing technology based on NGS is able to demonstrate the genetic diversity within cancer cell clones at the resolution of single cell [58]. Based on the subclones information from single cell sequencing technology, the process of cancer metastasis could be precisely reconstructed. Thus, it is expected to reveal the subclones carrying metastatic mutations and identify the clinical biomarker for metastasis treatment by single cell sequencing technology.

\section{The Prospect of Personalized Therapeutics by NGS}

Patients have benefited a lot from targeted cancer therapy and consequently avoided the harmful side effects of previous cancer therapies such as chemotherapy. However, a large subset of patients

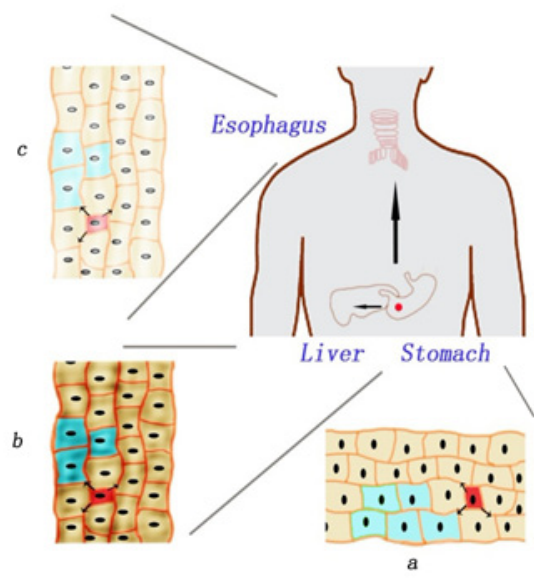

Figure 1: The cancer cell clones in primary stomach tumor (a), metastatic live tumor (b) and metastatic esophagus tumor (c). The main tumor subclones are marked in yellow. The different level of yellow in primary and metastatic tumors means the genetic diversity within the subclonal. The red represents the dominate subclone whereas the other color means the minor subclones. The figure indicates that metastatic tumour may arise from the cells within the primary tumour. still suffers from a certain cancer type, since they don't have good response to targeted cancer therapy. If each patient is expected to be cured, it is critical to shift the therapeutic strategy from targeted therapy to personalized therapeutics. To achieve the goal, it is necessary to identify specific biomarkers for each subset of patients. The increased understanding of cancer genome by NGS has laid the underlying basis for personalized therapeutics. Thus, NGS technologies provide the possibility to develop the special clinical test for each individual so as to achieve personalized therapeutics. Generally, correct diagnosis is the first and critical step for good therapeutic response. As the first step of personalized therapeutics, it is most potential to identify individually diagnostic biomarkers for prevalent cancer types. Such biomarkers can solve the problem of high false positive in diagnosis, which is originated from traditional methods, e. g. histopathology.

Blood is very suitable to serve as the source of personalized therapeutics because it is easy and noninvasive to obtain from individuals. Circulating tumor cells (CTCs) in peripheral blood from cancer patients, identified in a wide range of malignancies, has already been used as the biomarker for diagnosis and prognosis $[59,60]$. CTCs have been found in solid tumors, such as prostate and lung cancer $[61,62]$. Cancers could give rise to CTCs that are central to the establishment of metastasis. The presence and prognostic significance of CTCs have been highlighted by recent researches in the patients with metastatic cancers $[62,63]$. These researches have demonstrated that CTCs are very promising as the prognostic biomarker. However, the sensitivity of CTCs is limited for common capturing methods by single marker. Thus, CTCs have limited availability as the diagnostic biomarker for early-stage cancers. But, the multimarker method could capture CTCs. It seems to potentially improve the availability of CTCs as the diagnostic biomarker. A recent research has demonstrated a three-marker method (CK19, hMAM and CEA) to detect CTCs in the patients with breast cancer [64]. The sensitivity of CTCs in earlystage breast cancer reached $58.8 \%$, showing the higher sensitivity of multimarker than single marker. Even if this is an obvious progress, the sensitivity is still not high enough in clinic. Recent researches have showed that NGS technologies are very promising in developing accurate clinical testing for personalized therapeutics $[65,66]$. The possibility of detecting CTCs in peripheral blood shows its potential of clinical use in personalized therapies. It could serve as the evidence of early-diagnosis and prognosis. If cancer genome sequencing gradually becomes affordable with decreasing price, NGS will greatly improve the personalized therapeutics. Other types of cancer-derived DNA in blood or body fluids, together with the development of more sensitive methods, are also expected to be identified by NGS technologies as effective biomarkers in personalized therapeutics.

Certain cancer genes have already been used to diagnose earlystage cancer patients and predict their response to therapies. Thus, the testing of specific mutant genes has already entered into the stage of clinical trials. However, the final purpose of cancer genome research is to identify all mutated genes in each cancer type for diagnosis, prognosis and treatment. Now, the clinical application is only restricted to prevalent cancer types and the limited cancer genes. It is still a long way to achieve personalized therapeutics for various cancer types in clinic. To accelerate the discovery of individualized biomarkers and achievement of personalized therapeutics, scientists with professional cancer research knowledge in the universities and institutes should collaborate with the clinicians in hospitals providing 
Citation: Li J, Wang B, Fang H, Yang X (2012) The Development of Cancer Genome Research and its Clinical Opportunity brought by Next Generation Sequencing. J Health Med Informat S1. doi:10.4172/2157-7420.S1-002

sufficient tumor tissue samples, as well as organizations offering NGS service. For example, the largest genome center in the world, Beijing Genomics Institute (BGI), has announced in the $6^{\text {th }}$ international conference on genomics that four thousand of cancer genomes have been sequenced in 2011. With collaborations of worldwide scientists, more cancer genomes are supposed to be sequenced in the coming several years. Furthermore, it is also essential to constitute the global cancer research association for international collaboration. In 2010, International Cancer Genome Consortium (ICGC) was launched to coordinate global collaborators to conduct research projects of 50 different cancer types and/or subtypes with clinical and societal importance around the world [67]. The primary goals of the ICGC are to generate comprehensive catalogues of genomic abnormalities by NGS technologies and make the data available to the entire research community for clinical application. It is expected that more international collaborations will promote the early achievement of personalized therapeutics. The complete set of causative cancer genes in each cancer type is hopeful to be identified in the near future. Their roles in tumorigenesis are also expected to be interpreted clearly. In this way, cancer genome sequencing will become a routine clinical testing for individuals to achieve personalized therapeutics.

\section{References}

1. Chabner BA, Roberts TG (2005) Timeline: Chemotherapy and the war on cancer. Nat Rev Cancer 5: 65-72.

2. Gerber DE (2008) Targeted therapies: a new generation of cancer treatments. Am Fam Physician 77: 311-319.

3. Futreal PA, Coin L, Marshall M, Down T, Hubbard T, et al. (2004) A census of human cancer genes. Nat Rev Cancer 4: 177-183.

4. Nowell PC (1976) The clonal evolution of tumour cell populations. Science 195: 23-28.

5. Nowell PC, Hungerford D (1960) A minute chromosome in chronic granulocytic leukemia. Science 142: 1497

6. Reddy EP, Reynold RK, Santos E, Barbacid M (1982) A point mutation is responsible for the acquisition of transforming properties of the T24 human bladder carcinoma oncogene. Nature 300: 149-152.

7. Tabin CJ, Bradley SM, Bargmann Cl, Weinberg RA, Papageorge AG, et al. (1982) Mechanism of activation of a human oncogene. Nature 300: 143-149.

8. Capon DJ, Seeburg PH, McGrath JP, Hayflick JS, Edman U, et al. (1983) Activation of Ki-ras2 gene in human colon and lung carcinomas by two different point mutations. Nature 304: 507-513.

9. McGrath JP, Capon DJ, Smith DH, Chen EY, Seeburg PH, et al. (1983) Structure and organization of the human Ki-ras proto-oncogene and a related processed pseudogene. Nature 304: 501-506.

10. Shimizu K, Birnbaum D, Ruley MA, Fasano O, Suard Y, et al. (1983) Structure of the Ki-ras gene of the human lung carcinoma cell line Calu-1. Nature 304: 497-500.

11. Bos JL, Toksoz D, Marshall CJ, Verlaan-de Vries M, Veeneman GH, et al. (1985) Amino-acid substitutions at codon 13 of the $\mathrm{N}$-ras oncogene in human acute myeloid leukaemia. Nature 315: 726-730.

12. Downward J (2003) Targeting RAS signalling pathways in cancer therapy. Nat Rev Cancer 3: 11-22

13. Davies H, Bignell GR, Cox C, Stephens P, Edkins S, et al. (2002) Mutations of the BRAF gene in human cancer. Nature 417: 949-954.

14. Flaherty KT, Puzanov I, Kim KB, Ribas A, McArthur GA, et al. (2010) Inhibition of mutated, activated BRAF in metastatic melanoma. N Engl J Med 363: 809819 .

15. Ciardiello F, Caputo R, Damiano V, Caputo R, Troiani T, et al. (2003) Antitumor effects of ZD6474, a small molecule vascular endothelial growth factor receptor tyrosine kinase inhibitor, with additional activity against epidermal growth factor receptor tyrosine kinase. Clin Cancer Res 9: 1546-1556.

16. Hirata A, Ogawa S, Kometani T, Kuwano T, Naito S, et al. (2002) ZD1839 (Iressa) induces antiangiogenic effects through inhibition of epidermal growth factor receptor tyrosine kinase. Cancer Res 62: 2554-2560.

17. Flaherty KT, Puzanov I, Kim KB, Ribas A, McArthur GA, et al. (2010) Inhibition of mutated, activated BRAF in metastatic melanoma. N Engl J Med 363: 809819.

18. Nazarian R, Shi H, Wang Q, Kong X, Koya RC, et al. (2010) Melanomas acquire resistance to B-RAF(V600E) inhibition by RTK or N-RAS upregulation. Nature 468: 973-977.

19. Johannessen CM, Boehm JS, Kim SY, Thomas SR, Wardwell L, et al. (2010) COT drives resistance to RAF inhibition through MAP kinase pathway reactivation. Nature 468: 968-972.

20. Allegra CJ, Jessup JM, Somerfield MR, Hamilton SR, Hammond EH, et al (2009) American Society of Clinical Oncology provisional clinical opinion: testing for KRAS gene mutations in patients with metastatic colorectal carcinoma to predict response to anti-epidermal growth factor receptor monoclonal antibody therapy. J Clin Oncol 27: 2091-2096.

21. Lièvre A, Bachet JB, Boige V, Cayre A, Le Corre D, et al. (2008) KRAS mutations as an independent prognostic factor in patients with advanced colorectal cancer treated with cetuximab. J Clin Oncol 26: 374-379.

22. Amado RG, Wolf M, Peeters M, Van Cutsem E, Siena S, et al. (2008) Wildtype KRAS is required for panitumumab efficacy in patients with metastatic colorectal cancer. J Clin Oncol 26: 1626-1634.

23. Eberhard DA, Johnson BE, Amler LC, Goddard AD, Heldens SL, et al. (2005) Mutations in the epidermal growth factor receptor and in KRAS are predictive and prognostic indicators in patients with non-small-cell lung cancer treated with chemotherapy alone and in combination with erlotinib. J Clin Oncol 23 5900-5909.

24. Santarius T, Shipley J, Brewer D, Stratton MR, Cooper CS (2010) A census of amplified and overexpressed human cancer genes. Nat Rev Cancer 10: 59-64

25. Mitelman F, Johansson B, Mertens F(2007) The impact of translocations and gene fusions on cancer causation. Nat Rev Cancer 7: 233-245.

26. Mitelman F, Johansson B, Mertens F (2004) Fusion genes and rearranged genes as a linear function of chromosome aberrations in cancer. Nat Genet 36: 331-334.

27. Kumar-Sinha C, Tomlins SA, Chinnaiyan AM (2008) Recurrent gene fusions in prostate cancer. Nat Rev Cancer 8: 497-511.

28. Koivunen JP, Mermel C, Zejnullahu K, Murphy C, Lifshits E, et al. (2008) EML4 ALK fusion gene and efficacy of an ALK kinase inhibitor in lung cancer. Clin Cancer Res 14: 4275-4283.

29. Deininger MW, Druker BJ(2003) Specific targeted therapy of chronic myelogenous leukemia with imatinib. Pharmacol Rev 55: 401-423.

30. Coiffier B, Lepage E, Briere J, Herbrecht R, Tilly H, et al. (2002) CHOP chemotherapy plus rituximab compared with $\mathrm{CHOP}$ alone in elderly patients with diffuse large-B-cell lymphoma. N Engl J Med 346: 235-242.

31. Motzer RJ, Hutson TE, Tomczak P, Michaelson MD, Bukowski RM, et al (2007) Sunitinib versus interferon alfa in metastatic renal-cell carcinoma. N Engl J Med 356: 115-124.

32. Scheinfeld N (2006) A comprehensive review of imatinib mesylate (Gleevec) for dermatological diseases. J Drugs Dermatol 5: 117-122.

33. Park JW, Kerbel RS, Kelloff GJ, Barrett JC, Chabner BA, et al. (2004) Rationale for biomarkers and surrogate end points in mechanism-driven oncology drug development. Clin Cancer Res 10: 3885-3896.

34. Partridge AH, Avorn J, Wang PS, Winer EP (2002) Adherence to therapy with oral antineoplastic agents. J Natl Cancer Inst 94: 652-661.

35. Cunningham D, Humblet $Y$, Siena S, Khayat D, Bleiberg H, et al. (2004) 
Citation: Li J, Wang B, Fang H, Yang X (2012) The Development of Cancer Genome Research and its Clinical Opportunity brought by Next Generation Sequencing. J Health Med Informat S1. doi:10.4172/2157-7420.S1-002

Page 8 of 8

Cetuximab monotherapy and cetuximab plus irinotecan in irinotecan-refractory metastatic colorectal cancer. N Engl J Med 351: 337-345

36. Copeland NG, Jenkins NA (2009) Deciphering the genetic landscape of cancer-from genes to pathways. Trends Genet 25: 455-462.

37. Sjöblom T, Jones S, Wood LD, Parsons DW, Lin J, et al. (2006) The consensus coding sequences of human breast and colorectal cancers. Science 314: 268274 .

38. Jones S, Zhang X, Parsons DW, Lin JC, Leary RJ, et al. (2008) Core signaling pathways in human pancreatic cancers revealed by global genomic analyses. Science 321: 1801-1806.

39. Parsons DW, Jones S, Zhang X, Lin JC, Leary RJ, et al. (2008) An integrated genomic analysis of human glioblastoma multiforme. Science 321: 1807-1812

40. Ley TJ, Mardis ER, Ding L, Fulton B, McLellan MD, et al. (2008) DNA sequencing of a cytogenetically normal acute myeloid leukaemia genome. Nature 456: 66-72.

41. Mardis ER, Ding L, Dooling DJ, Larson DE, McLellan MD, et al. (2009) Recurring mutations found by sequencing an acute myeloid leukemia genome. N Engl J Med 361: 1058-1066.

42. Ley TJ, Ding L, Walter MJ, McLellan MD, Lamprecht T, et al. (2010) DNMT3A mutations in acute myeloid leukemia. N Engl J Med 363: 2424-2433.

43. Paschka P, Schlenk RF, Gaidzik VI, Habdank M, Krönke J, et al. (2010) IDH1 and IDH2 mutations are frequent genetic alterations in acute myeloid leukemia and confer adverse prognosis in cytogenetically normal acute myeloid leukemia with NPM1 mutation without FLT3 internal tandem duplication. J Clin Oncol 28 : 3636-3643.

44. Link DC, Schuettpelz LG, Shen D, Wang J, Walter MJ, et al. (2011) Identification of a novel TP53 cancer susceptibility mutation through whole-genome sequencing of a patient with therapy-related AML. JAMA 305: 1568-1576.

45. Patel KP, Ravandi F, Ma D, Paladugu A, Barkoh BA, et al. (2011) Acute myeloid leukemia with IDH1 or IDH2 mutation: frequency and clinicopathologic features. Am J Clin Pathol 135: 35-45.

46. Boissel N, Nibourel O, Renneville A, Gardin C, Reman O, et al. (2010) Prognostic impact of isocitrate dehydrogenase enzyme isoforms 1 and 2 mutations in acute myeloid leukemia: a study by the Acute Leukemia French Association group. J Clin Oncol 28: 3717-3723.

47. Paschka P, Schlenk RF, Gaidzik VI, Habdank M, Krönke J, et al. (2010) IDH1 and IDH2 mutations are frequent genetic alterations in acute myeloid leukemia and confer adverse prognosis in cytogenetically normal acute myeloid leukemia with NPM1 mutation without FLT3 internal tandem duplication. J Clin Oncol 28 3636-3643.

48. Stratton MR, Campbell PJ, Futreal PA (2009) The cancer genome. Nature 458 719-724.

49. Greenman C, Stephens P, Smith R, Dalgliesh GL, Hunter C, et al. (2007) Patterns of somatic mutation in human cancer genomes. Nature 446: 153-158.

50. Lee W, Jiang Z, Liu J, Haverty PM, Guan Y, et al. (2010) The mutation spectrum revealed by paired genome sequences from a lung cancer patient. Nature 465 : 473-437

51. Pleasance ED, Cheetham RK, Stephens PJ, McBride DJ, Humphray SJ, et al. (2010) A comprehensive catalogue of somatic mutations from a human cancer genome. Nature 463: 191-196.

52. Pleasance ED, Stephens PJ, O'Meara S, McBride DJ, Meynert A, et al. (2010) A small-cell lung cancer genome with complex signatures of tobacco exposure. Nature 463: 184-190

53. Shah SP, Morin RD, Khattra J, Prentice L, Pugh T, et al. (2009) Mutationa evolution in a lobular breast tumour profiled at single nucleotide resolution. Nature 461: 809-813.

This article was originally published in a special issue, Clinical Translational Research handled by Editor(s). Dr. Carolyn Estes-Rodgers, Governors State University, USA
54. Campbell PJ, Stephens PJ, Pleasance ED, O'Meara S, Li H, et al. (2008) Identification of somatically acquired rearrangements in cancer using genomewide massively parallel paired-end sequencing. Nature Genetics 40: 722-729.

55. Stratton MR(2011) Exploring the genomes of cancer cells: progress and promise. Science 331: 1553-1558.

56. Ding L, Ellis MJ, Li S, Larson DE, Chen K, et al. (2010) Genome remodelling in a basal-like breast cancer metastasis and xenograft. Nature 464: 999-1005.

57. Tie J, Lipton L, Desai J, Gibbs P, Jorissen RN, et al. (2011) KRAS mutation is associated with lung metastasis in patients with curatively resected colorectal cancer. Clin Cancer Res 17:1122-1130.

58. Navin N, Kendall J, Troge J, Andrews P, Rodgers L, et al. (2011) Tumour evolution inferred by single-cell sequencing. Nature 472: $90-94$

59. Takao M, Takeda $\mathrm{K}(2011)$ Enumeration, characterization, and collection of intact circulating tumor cells by cross contamination-free flow cytometry. Cytometry A 79: 107-117

60. Zhe X, Cher ML, Bonfil RD (2011) Circulating tumor cells: finding the needle in the haystack. Am J Cancer Res 1: 740-751.

61. Panteleakou Z, Lembessis P, Sourla A, Pissimissis N, Polyzos A, et al. (2009) Detection of circulating tumor cells in prostate cancer patients: methodological pitfalls and clinical relevance. Mol Med 15: 101-114.

62. Lecharpentier A, Vielh P, Perez-Moreno P, Planchard D, Soria JC, et al. (2011) Detection of circulating tumour cells with a hybrid (epithelial/mesenchymal) phenotype in patients with metastatic non-small cell lung cancer. $\mathrm{Br} \mathrm{J}$ Cancer 105: 1338-1341.

63. Gradilone A, Naso G, Raimondi C, Cortesi E, Gandini O, et al. (2011) Circulating tumor cells (CTCs) in metastatic breast cancer (MBC): prognosis, drug resistance and phenotypic characterization. Ann Oncol 22: 86-92.

64. Chen Y, Zou TN, Wu ZP, Zhou YC, Gu YL, et al. (2010) Detection of cytokeratin 19 , human mammaglobin, and carcinoembryonic antigen-positive circulating tumor cells by three-marker reverse transcription-PCR assay and its relation to clinical outcome in early breast cancer. Int J Biol Markers 25: 59-68.

65. Leary RJ, Kinde I, DiehI F, Schmidt K, Clouser C, et al. (2010) Development of personalized tumor biomarkers using massively parallel sequencing. Sci Trans Med 2: 20ra14

66. McBride DJ, Orpana AK, Sotiriou C, Joensuu H, Stephens PJ, et al. (2010) Use of cancer-specific genomic rearrangements to quantify disease burden in plasma from patients with solid tumors. Genes Chromosomes Cancer 49: 1062-1069.

67. International Cancer Genome Consortium (2010) International network of cancer genome projects. Nature 464: 993-998. 